\title{
Primary Bipolar Arthroplasty in Unstable Intertrochanteric Fractures in Elderly
}

\author{
Ahmed Elmorsy ${ }^{1}$, Mahmoud Saied ${ }^{2}$, Adel Awad Allah ${ }^{2}$, Mahmoud Zaied $^{2}$, Mahmoud Hafez $^{1}$ \\ ${ }^{1}$ Orthopaedic Department, October 6 University, Cairo, Egypt; ${ }^{2}$ Orthopaedic Department, Alazhar University, Cairo, Egypt. \\ Email: ah_morsy@yahoo.com
}

Received December $18^{\text {th }}, 2011$; revised January $24^{\text {th }}, 2012$; accepted February $8^{\text {th }}, 2012$

\begin{abstract}
Unstable intertrochanteric fracture in elderly is a challenging surgical condition with a high risk of morbidity and mortality. The aim of this study is to evaluate the results of bipolar arthroplasty as an alternative to the standard treatment of internal fixation. Materials and Methods: 41 patients (22 females and 19 males - all were 65 years old or above) who had bipolar arthroplasty for unstable intertrochanteric fractures were prospectively evaluated. Modified lateral approach was used in all patients. Harris Hip Score (HHS) used for clinical evaluation, however radiological follow up was done using plain X-rays. Follow up period ranged from 12 to 24 months. Results: During the last follow up, the Harris Hip Score ranged from 93 to 51 with a mean value 78.19 . Four cases $(9.76 \%)$ were excellent $(91-100), 16$ cases $(39.02 \%)$ good $(81-90), 16$ cases $(39.02 \%)$ fair $(71-80)$ and 5 cases $(12.02 \%)$ poor $(\leq 70)$. Six cases had complications; infection (1), dislocation (1), stem loosening and subsidence (2), bleeding peptic ulcer (1) and intra-operative crack of the femur while preparing the femoral canal for implant insertion (1). Five patients died within the first year (12.19\%). Four patients had revision surgery. Conclusion: Bipolar hemiarthroplasty for the unstable intertrochanteric fractures of the femur in elderly has good early clinical outcome. It is to be considered as one of the treatment options for such injury speciality in case of fragility fractures.
\end{abstract}

Keywords: Bipolar; Unstable; Intertrochanteric Fractures; Hemiarthroplasty

\section{Introduction}

Fractures of the proximal femur in elderly patients are generally caused by a single fall and is more common in elderly females [1]. Unstable intertrochanteric fractures in elderly constitute one of the major reasons for morbidity in this age group [2]. People in this age group usually have other systemic complications such as diabetes and cardiovascular diseases. The impacts of these diseases leads to a rapid deterioration in the general condition of these patients if they are kept bed ridden. The main goals for the treatment of these fractures in elderly patients are, to restore the pre-fracture activity status, to allow early full weight bearing and to avoid possible re-operation [3].

As a general rule, preservation of the patient's own bones is the ideal aim for the surgeons. In osteoporotic elderly patients with unstable intertrochanteric fracture this ideal aim will not bring the patient back his activity if internal fixation was done. Weak purchase of the internal fixation device due to osteoporosis and com- minution of the fracture increases the incidence of failure of internal fixation such as, cutting out the screws and displacement of the bone fragments [2]. We cannot rely on internal fixation devices to allow early full weight bearing of patient in presence of severe osteoporosis and marked comminution at the fracture site. Partial weight bearing is very difficult to be followed by these patients, so they shift to full weight bearing on the operated limb causing mechanical failure [2]. This prospective study was conducted to evaluate the results of bipolar arthroplasty for elderly patients with unstable intertrochanteric fractures.

\section{Patients and Method}

During the period of 3 years; a prospective study was done on 41 patients who got unstable intertrochanteric fractures. The inclusion criteria were: 1) Unstable intertrochanteric fractures (Figure 1), 2) Elderly patient (65 years or older), 3) Osteoporosis (Singh index from 4, 3, 2 \& 1). Patients with evidence of bone softening disease, inflammatory arthropathy \& who were non mobile preinjury were excluded from the study. An operation of cemented bipolar hemiarthroplasty was performed for all of these patients. Follow up period ranged from $12-24$ months with the mean follow up period 13.68 months. 36 out of the 41 patients completed the follow up period. 


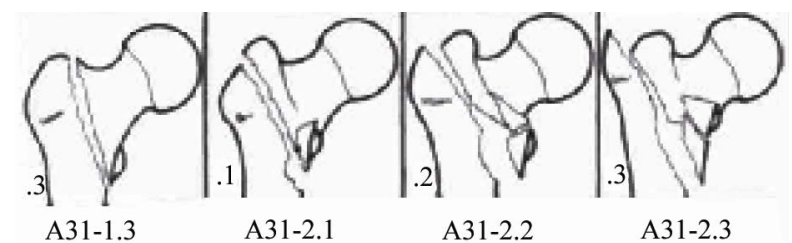

Figure 1. Fracture patterns which is included in this study, with code for each fracture according to the AO classification (A31-1.3, $\cdots)$.

All patients received 3rd generation cephalosporin's as antibiotics, low molecular weight heparin (LMWH), morphine for analgesia and proton pump inhibitor for protection against stress ulcers. In addition to that, the following radiological assessment was performed: AP view for the pelvis and hips radiograph with the Patient lying flat on the table and the lower extremities Internally rotated $15^{\circ}$. Careful preoperative templating of appropriate radiographs helped in restoring hip biomechanics.

Anaesthesia varied between general and regional (spinal and epidural) depending on the patient's general condition. General anaesthesia: 12 patients (29.27\%), Spinal anaesthesia: 13 Patients (31.71\%), and epidural anaesthesia: 16 patients $(39.02 \%)$. As for the surgical approach, the modified lateral (Harding) approach was used for all patients (with the patients in the lateral position). Clinical follow up was done after 2 weeks of discharge then every 3 months for the first 6 months then every 6 months for the following year. Harris hip score was used for clinical evaluation. Results were rated as excellent (91 - 100 points), good (81 - 90 points), fair (71 - 80 points) and poor ( $\leq 70$ points).

\section{Results}

The mean hospital stay period was 8.78 days (range 6 15 days). The mean interval time between the time of injury and the day of operation is 2.9 days. The mean intra-operative blood loss was $567 \mathrm{cc}$ (range 350 - 1000 cc). Post operative mortality: None of the patient died within the first 60 days. Five patients died within the first year $(12.19 \%)$. In four patients the causes of death was complications cardiac disease. However, there was no information about the cause of death for the 5 th patient who died 8 month post operative. Four patients were reoperated: revision due to loosening and subsidence (2 patients), open reduction for dislocated prothesis after failed closed reduction (1 patient), and revision due to infection (1 patient). Post-operatively 27 cases were able to ambulate independently using walker, whereas 13 cases were able to ambulate using walker but with assistance. At last follow up, the Harris Hip Score ranged from 93 to 51 with a mean value of 78.19 . Four cases $(9.76 \%)$ rated excellent $(91-100), 16$ cases $(39.02 \%)$ good (81 - 90), 16 cases (39.02\%) fair $(71-80)$ and 5 cases $(12.02 \%)$ poor $(\leq 70)$ (Figure 2). According to Gruen scoring for cementation: 15 cases $(36.59 \%)$ scored A, 14 cases $(34.15 \%)$ B, 2 cases $(04.88 \%)$ C1, 5 cases $(12.20 \%) \mathrm{C} 2$ and 5 cases $(12.20 \%) \mathrm{D}$.

Complications: In this study, six cases suffered from different complications: Infection (1 patient), Dislocation (1 patient), stem subsidence (1 patients), acetabular wear (1 patient) post operative bleeding peptic ulcer (1 patient) and intra-operative crack during the insertion of the femoral stem (1 patient) with weight bearing delayed for 6 weeks for this particular case (Figures 3 and 4).

\section{Discussion}

Several surgical options exist for the treatment of unstable intertrochanteric fractures. Traditionally, the consensus was to preserve the normal bone by open reduction and internal fixation. The technique is familiar to orthopaedic surgeons, and it is relatively rapid [4]. Arthroplasty is a less frequently employed alternative, although it allows immediate full weight bearing. Many of the complications of internal fixation (e.g. non-union) are avoided by performing arthroplasty [4]. Several studies have been published reporting the results of treatment using different techniques. Studies of internal fixation of both stable and unstable intertrochanteric hip fractures reported failure rate between $6 \%-32 \%$ [2,4-6]. Theoretically, bipolar hemiarthroplasties were introduced to address the complications of unipolar implants like acetabular wear, protrusio, loosening, and dislocation. Stems

$$
\begin{aligned}
& \text { Excellent } \\
& 9.76 \%
\end{aligned}
$$

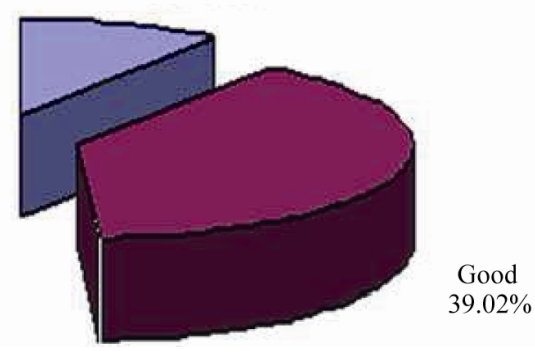

Figure 2. Results at last follow up according to Harris Hip Score. 


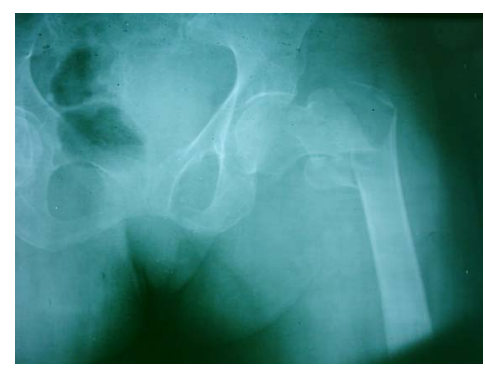

Figure 3. Pre-operative X-ray.

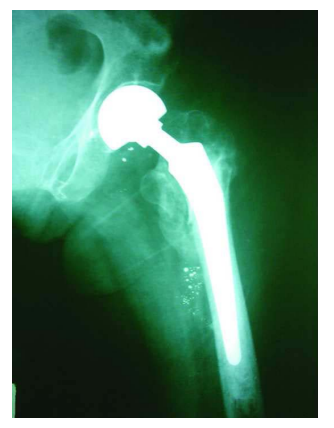

Figure 4. X-ray at last follow up 12th month post operation.

were reconfigured, more in line with total hip replacement designs, to lessen component loosening. Inner bearing motion was introduced to reduce acetabular wear and dislocation rates. Modularity allowed for sizing to improve stability [7]. In unstable intertrochanteric fractures management; the choice of bipolar hemiarthroplasty prosthesis raised a new question: which stem design should be used? The deficient proximal medial femur is one of the challenges faced during surgery. It is either to be augmented with calcar replacement prosthesis, or the calcar has to be reconstructed. Several investigations have reported a good to excellent functional results with the used of calcar replacement femoral prosthesis [4].

By comparing the results of this study with the results obtained by Faldini [5], Chris [4], Haentjens [2], Rady [6] and others it was found that: In this study the mean age group was 72.51 years. While in Faldini's, Chris's, Rady's and Haentjens's study, the mean age was $81,80,85.04$ and 82 years old respectively. Hence, this study targeted the same age group as in other studies. The mean Harris Hip Score in this study at the last follow up was 78.19, with about $50 \%$ of the patients were enjoying excellent to good results and only $12 \%$ had poor results. In comparison to our results in Haentjens's [2] series about $78 \%$ of the patients got excellent to good results. In Rady's [6] study (who used Merle d, Aubigne as a scoring system) about $63 \%$ of the patients got excellent to good results.

One of the reasons that may affect the outcome of the operation is the time interval trauma to and surgery which was 2.9 days in this study, 2.81 days in Rady's [6] study, while in Faldini's [5] study all the patients were operated within the first 48 hours of the trauma resulting in $0 \%$ of dislocation, $0 \%$ of loosening or infection with mean HHS at 1 year was 76 . We tried to shorten this time interval. About $41.46 \%$ of the patients had their operation delayed for preoperative workup and optimisation due to other systemic diseases. In addition, there were other non-medical reasons for the delay such as financial issues and the availability of implants. We had no inhospital mortality, however, 1 year mortality rate was $12.19 \%(5 / 41)$. In Haentjens's [2] study the 1 year mortality rate was $35 \%$. This could be explained by the fact that the mean age in his study was 82 years, whereas the mean age in our study was 72.51 years. In Chris's [4] study the 1 year mortality age was $10.3 \%$ and the mean age was 80 years. In Rady's [6] study the one year mortality was $18.75 \%$ with the mean age being 85.04 years. In Faldini's [5] study, despite the mean age being 81 years, the 1 year mortality was $19 \%$. Furthermore, all the operations in Faldini's study were performed within 48 hours from the trauma. It may be that this time interval from the trauma till the operation may have an effect to get this mortality rate in this high age group. In different studies, the hospital mortality rate for unstable intertrochanteric fractures treated by open reduction and internal fixation ranged from $4 \%-17 \%$ [2,8-10]. However, it was from 0 to $7.3 \%$ for patients treated by bipolar hemiarthroplasty the in hospital $[2,4,6,11-14]$. This could be referred to the early post operative ambulation later group.

In our series there were 4 cases $(9.7 \%)$ of re-operation; one case for infection, one case for subsidence, and one case for dislocation where an open reduction was done and one for acetabular wear. In Rady's study [6]; the rate of re-operation was $4.1 \%$; 1 case for infection that necessitates implant removal and one case of dislocation which was managed by open reduction. There was no failure or re-operation in Faldini's study [5], however Haentjens's re-operated on $5.4 \%$ of the patients [2]. In this study the average blood loss was $567 \mathrm{cc}$. which was higher than the reported blood loss value in Faldini's study [5] (247 cc.) and in Chris's study (475 cc). Failure and re-operation which is highly dependent on the prefracture activity level. In Chris's study [4] there were $66.66 \%$ of the patients who were able to ambulate in-dependently; and they got improved over the next year (they were independent ambulant pre-fracture). Only $33.33 \%$ were able to walk with assistance and they didn't improve at 1 year follow up. All the patients in their study were ambulant postoperatively. In this study 26 cases $(63 \%)$ out of the 41 cases were able to ambulate (pre-discharge) using walkers, only 14 cases (34\%) were in need for help of another person. Only one case was not able to ambulate pre-discharge as there were a crack in the femur that happened intra-operative; so we decided to 
delay the weight bearing till the fracture heals. There was no postoperative general complication like-chest infection, Deep Venous Thrombosis (DVT) and pulmonary embolism. There was one case of postoperative bleeding peptic ulcer and he was admitted to the ICU for 2 days after which he was improved. This condition highlights the importance of giving the patients peri-operative medication (Proton pump inhibitor) for gastric protection against stress ulcers, especially in the elderly. In Chris's study [4], there were only one case of DVT and 2 cases of pressure sore for 2 patients who were not ambulant postoperatively. Postoperative ambulation is one of the goals of this method of management for this type of fracture. In this study; one patient with no history of peptic ulcer developed hematemesis on the day of surgery, and he was admitted to the ICU for 2 days after which he was improved. This condition highlights the importance of giving the patients peri-operative medication (Proton pump inhibitor) for gastric protection against stress ulcers, especially in the elderly.

In another case an intra-operative crack of the femur was occurred. It was un-displaced and managed by cerclage wire. Postoperative weight bearing was delayed for 6 weeks. This patient was died 11 months postoperative from complications of liver disease. This study did not take into consideration the preoperative acetabular conditions such as osteoarthritics, which may affect clinical outcomes of hemiarthroplasty. Another limitation is the short-term follow.

\section{Conclusion}

Hemiarthroplasty using bipolar prostheses for the unstable intertrochanteric fractures of the femur in elderly has good clinical results; in terms of early post-operative ambulation. This will have a direct effect on the general condition and the post-operative rehabilitation. Patient selection is very important as we are directing this to the elderly people with sub-normal bone quality having unstable intertrochanteric fractures. So, bipolar hemiarthroplasty should be considered as one of the modalities of the treatment of unstable intertrochanteric fractures in elderly.

\section{REFERENCES}

[1] S. I. Greenspain, F. Myers and L. A. Maintland, "Fall Severity and Bone Mineral Density as Risk Fractures for Hip Fracture in Ambulatory Elderly," JAMA, Vol. 271, No. 2, 1994, pp. 128-133. doi:10.1001/jama.1994.03510260060029

[2] P. Haentjens, P. P. Casteleyn, H. De Boek, F. Handelberg and P. Opdecam, "Treatment of Unstable Intertrochanteric and Subtrochanteric Fractures in Elderly. Primary Bipolar Arthroplasty Compared with Internal Fixation," Journal of Bone and Joint Surgery, Vol. 71, No. 8, 1989, pp. 1214-
1225.

[3] O. Rodop, A. Kiral, H. Kaplan and I. Akmaz, "Primary Bipolar Hemiprosthesis for Unstable Intertrochanteric Fractures," International Orthopaedics, Vol. 26, No. 4, 2002, pp. 233-237. doi:10.1007/s00264-002-0358-0

[4] C. Grimsrud, R. J. Monzon, J. Richman and M. D. Ries, "Cemented Hip Arthroplasty with a Novel Cerclage Cable Technique for Unstable Intertrochanteric Hip Fractures," The Journal of Arthroplasty, Vol. 20, No. 3, 2005, pp. 337-343.

[5] C. Faldini, G. Grandi, M. Romagnoli, S. Pagkrati, V. Digennaro, O. Faldini and S. Giannini, "Surgical Treatment of Unstable Intertrochanteric Fractures by Bipolar Hip Replacement or Total Hip Replacement in Elderly Osteoporotic Patients," Journal of Orthopaedics and Traumatology, Vol. 7, No. 3, 2006, pp. 117-121. doi:10.1007/s10195-006-0133-X

[6] A. E. Rady, A. A. Sharaf and A. A. Abuelela, "Primary Bipolar Hemiarthroplasty in Unstable Intertrochanteric Fractures in Elderly," 24th SICOT Meeting (International Society for Orthopaedic Surgery and Traumatology), Cairo, 2003.

[7] R. A. Wathne, K. J. Koval, G. B. Aharonoff and J. D. Zukerman, "Modular Unipolar versus Bipolar: A Prospective Evaluation of Functional Outcome after Femoral Neck Fracture," Journal of Orthopaedic Trauma, Vol. 9, No. 4, 1995, pp. 298-302. doi:10.1097/00005131-199509040-00005

[8] R. F. Kyle, R. B. Gustillo and R. F. Premer, "Analysis of Six Hundered and Twenty Two Intertrochanteric Hip Fractutes," Journal of Bone \& Joint Surgery, Vol. 61, No. 2, 1979, pp. 216-221.

[9] D. C. R. Hardy, P.-Y. Descamps, P. Krallis, L. Fabeck, P. Smets, C. L. Bertens and P. E. Delince, "Use of an Intramedullary Hip-Screw Compared with a Compression Hip-Screw with Plate for Intertrochanteric Femoral Fractures. A Prospective, Randomized Study of One Hundred Patients," Journal of Bone \& Joint Surgery, Vol. 80, No. 5, 1998, pp. 618-630.

[10] G. J. T. Haidukewych, T. A. Israel and D. J. Berry, et al., "Reverse Obliquity Fractures of Intertrochanteric Region of the Femur," Journal of Bone \& Joint Surgery, Vol. 83, No. 5, 2001, pp. 643-650.

[11] S. F. Harwin, R. E. Stern and R. G. Kulick, "Primary bateman-Leinbach Bipolar Prosthesis Replacement at the Hip in the Treatment of Unstable Intertrochanteric Fractures in Elderly," Orthopaedics, Vol. 13, No. 10, 1990, pp. 1131-1136.

[12] S. Green, T. Moor and F. Proano, "Bipolar Prosthesis Replacement for the Management of Unstable Intertrochanteric Hip Fractures in Elderly," Clinical Orthopaedics and Related Research, Vol. 224, 1987, pp. 169-177.

[13] K. C. Chan and G. S. Gill, "Cemented Hemiarthroplasty for Elderly Patients with Intertrochanteric Fractures," Clinical Orthopaedics and Related Research, Vol. 371, pp. 206-215. doi:10.1097/00003086-200002000-00025

[14] M. B. Stern and A. Angerman, "Comminuted Intertrochanteric Fractures Treated with a Leinbach Prosthesis," Clinical Orthopaedics and Related Research, Vol. 218, 1987, pp. 75-80. 
[15] R. F. Kyle, R. B. Gustillo and R. F. Premer, "Analysis of Six Hundered and Twenty Two Intertrochanteric Hip Fractutes," Journal of Bone \& Joint Surgery, Vol. 61, No. 2, 1979, pp. 216-221.

[16] D. C. R. Hardy, P.-Y. Descamps, P. Krallis, L. Fabeck, P. Smets, C. L. Bertens and P. E. Delince, "Use of an Intramedullary Hip-Screw Compared with a Compression Hip-Screw with Plate for Intertrochanteric Femoral Frac- tures. A Prospective, Randomized Study of One Hundred Patients," Journal of Bone \& Joint Surgery, Vol. 80, No. 5, 1998, pp. 618-630.

[17] G. J. T. Haidukewych, T. A. Israel and D. J. Berry, et al., "Reverse obliquity fractures of intertrochanteric region of the Femur," Journal of Bone \& Joint Surgery, Vol. 83, No. 5, 2001, pp. 643-650. 